Ambulatório de Endocrinologia, Hospital Universitário da Universidade Federal de Santa Catarina (UFSC), Florianópolis, SC, Brasil ${ }^{2}$ Núcleo de Pesquisas em Neurologia Experimental e Clínica (Nupnec), UFSC Florianópolis, SC, Brasil
Correspondência para: Alexandre Hohl

Av. Rio Branco, 404/704, torre 1 Centro

88015-200 - Florianópolis, SC, Brasil alexandrehohl@endocrino.org.br

Recebido em 31/Ago/2009 Aceito em 1/Out/2009

\title{
Avaliação tardia do eixo hipofisário-gonadal em pacientes adultos que sofreram traumatismo cranioencefálico grave
}

\author{
Late evaluation of the pituitary-gonadal axis in \\ survivors of severe traumatic brain injury
}

\begin{abstract}
Alexandre Hohl',2, Jordana Bernardi Daltrozo', Camila Girardi Pereira', Thaís Rossoni Weber', Heyde Francine Pinto², Jackson da Silva Gullo², Juçara Deitos Bernardini², Marisa Helena César Coral ${ }^{1}$, Roger Walz ${ }^{2}$
\end{abstract}

\begin{abstract}
RESUMO
Objetivo: Avaliar a função hipofisária-gonadal nos pacientes vítimas de TCE graves ocorridos na Grande Florianópolis, entre 2000 e 2004. Métodos: Foram estudados 30 pacientes, sendo 22 homens e 8 mulheres, submetidos à avaliação clínica e laboratorial em seguimento médio de 4 anos após a data do traumatismo. Resultados: Os homens possuíam em média 38 anos no ano da avaliação, enquanto as mulheres, 42 anos. A maioria dos traumatismos está relacionada aos acidentes de trânsito (63,3\%). Três pacientes (10\%) estavam com valores de FSH abaixo do normal e apenas 1 paciente (3,3\%) apresentou LH alterado. Nas mulheres avaliadas, os níveis de estradiol foram normais. Na população masculina, foi evidenciado nível de testosterona baixo em 2 pacientes $(9,1 \%)$. Todos os pacientes apresentavam normoprolactinemia. Conclusão: Dois casos de hipogonadismo masculino $(9,1 \%)$ foram diagnosticados neste estudo. Isso indica a necessidade de atenção aos pacientes sobreviventes deTCE grave para realizar diagnóstico precoce de hipogonadismo. Arq Bras Endocrinol Metab. 2009;53(8):1012-19
\end{abstract}

Descritores

Traumatismo cranioencefálico; hipogonadismo; hipopituitarismo; testosterona

\begin{abstract}
Objective: The purpose of this study is to evaluate pituitary function impairment in order to verify the prevalence of sex hormone deficiency and to analyze the profile of TBI population. Methods: Thirty patients were studied, 22 were male and 8 were female. All patients had their gonadal function assessed and they were evaluated at a median of 4 years post-trauma. Results: The average age of the men was 38 years at the time of the evaluation, while the mean age of women was 42 years. The majority of TBI was related to traffic accidents $(63.3 \%)$. Three patients (10\%) had low FSH and only 1 patient (3.3\%) had low LH. There was no biochemical evidence of hypogonadism in women. Two male patients presented low testosterone $(9.1 \%)$ and were diagnosed with hypogonadism. Prolactin levels were normal in all patients. Conclusion: Two cases of hypogonadism $(9.1 \%)$ were diagnosed among men in this study. It is therefore necessary that medical professionals involved in the management of TBI patients are aware of hypogonadism as a complication of TBI, in order to diagnose it early. Arq Bras Endocrinol Metab. 2009;53(8):1012-19
\end{abstract}

\section{Keywords}

Traumatic brain injury; hypogonadism; hypopituitarism; testosterone

\section{INTRODUÇÃO}

$\mathrm{O}$ traumatismo cranioencefálico (TCE) é uma das principais causas de morte e incapacidade em adultos jovens, com consequências que abrangem desde incapacidade física a deficiências cognitivas, comporta- mentais, psicológicas e sociais a longo prazo (1-3), o que torna o TCE um problema de saúde pública (4).

A incidência do TCE nos países desenvolvidos é de aproximadamente 200/100.000 (5). No Brasil, Masini (6) estimou a incidência de 314/100.000 habitantes 
no ano de 1991 em Brasília. Sabe-se, no entanto, que a real incidência de traumatismos é subestimada tanto pela não assistência médica aos casos leves quanto pela evolução desfavorável dos traumatismos severos antes mesmo do socorro médico.

Em diferentes estudos, confirma-se o predomínio do adulto jovem entre as vítimas de $\operatorname{TCE}(7,8)$, principalmente abaixo de 35 anos (9), fato que está relacionado com acidentes e violências, atingindo a faixa populacional de maior atividade laborativa (10). Os homens são mais acometidos, com taxa de incidência correspondente quase ao dobro daquela referente às mulheres. Em idades mais avançadas, essa relação se equivale, pelo aumento do número de quedas, cujos riscos são semelhantes em ambos os sexos (5).

O TCE está implicado em uma série de complicações tanto neurológicas quanto de outros aparelhos e sistemas. Entre elas, destacam-se disfunções cognitivas, alteração das percepções táteis e de outros sentidos e disfunção sexual, ocasionando mudanças comportamentais e implicações para as atividades cotidianas desses pacientes $(1,11,12)$.

Recentemente, o TCE tem sido reconhecido como frequente causa de disfunção hipotalâmica-hipofisária (13-15). Entre as disfunções pós-traumáticas vistas no eixo hipotálamo-hipofisário, encontra-se o hipopituitarismo. Este se refere a uma insuficiência parcial ou total da hipófise anterior em secretar seus hormônios (16), demonstrada bioquimicamente pela deficiência em, pelo menos, um eixo endócrino (17).

O hipopituitarismo pós-traumático pode ser dividido em alterações funcionais durante a fase aguda após TCE, que resultam em aumento ou decréscimo temporário das concentrações séricas hormonais, e alterações na secreção de hormônios hipofisários em qualquer momento após TCE, levando a um hipopituitarismo definitivo causado por lesão hipofisária e/ou hipotalâmica.

O quadro clínico é variável, compreendendo desde formas subclínicas, diagnosticadas apenas por meio de testes de estímulos, a situações que podem ameaçar a vida do paciente principalmente na fase aguda pós-TCE (13).

Muitos sintomas do hipopituitarismo podem assemelhar-se a consequências próprias do TCE (Tabela 1), o que explica o frequente atraso no diagnóstico do hipopituitarismo pós-traumático, significando uma perda na oportunidade de tratamento desses pacientes que teriam potencialmente uma melhor recuperação pós-TCE.

Por outro lado, é bem estabelecido que a resposta fisiológica da doença crítica e aguda inclui alterações
Tabela 1. Consequências do traumatismo cranioencefálico

\section{Disfunção neurológica (motora, sensorial e autonômica)}

Disfunção motora: paralisia, movimentos involuntários, ataxia

Espasticidade

Distúrbios visuais

Perda sensorial: paladar, tato, olfato, audição

Distúrbio da fala: afasia, disartria

Distúrbio do sono: insônia, fadiga

Disfunção sexual

\section{Disfunção cognitiva}

Perda de memória: dificuldades com novo aprendizado, diminuição da atenção e concentração; velocidade de pensamento diminuída; dificuldade em resolver problemas

Dificuldade em planejar, organizar e tomar decisões

Problemas na linguagem: disfasia, dificuldade em achar as palavras, alteração da escrita e da leitura

\begin{tabular}{l}
\hline Alterações comportamentais \\
\hline Distúrbios emocionais, labilidade emocional \\
Distúrbios psiquiátricos: ansiedade, depressão, estresse pós-traumático, \\
psicose \\
Apatia \\
Autoestima reduzida \\
Autocontrole reduzido: irritabilidade, impulsividade, desinibição \\
\hline Consequências no cotidiano \\
\hline Perda da independência \\
Desemprego \\
Dificuldades em manter relações interpessoais \\
\hline
\end{tabular}

hormonais, na maioria dos pacientes mimetizando hipotireoidismo e hipogonadismo central, podendo ser difícil distinguir entre alteração pós-traumática ou pela doença aguda ou crítica.

Sabe-se, hoje, que o hipopituitarismo pós-TCE é mais comum do que se pensava (18), porém permanece subdiagnosticado em muitos pacientes. Pelo menos uma disfunção neuroendócrina pode ser encontrada em $35 \%$ a $50 \%$ de pacientes com TCE $(13,19,20)$. Segundo Bondanelli e cols. (17), estudos publicados recentemente mostram uma prevalência de $30,1 \%$ para deficiência de hormônio de crescimento; $28,8 \%$ para deficiência de gonadotropinas; $18,5 \%$ para deficiência de adrenocorticotropinas e de tireotropinas. Essas disfunções podem ocorrer precocemente e com alta frequência, porém a instalação destas também pode se dar tardiamente, de meses a anos após a lesão, sendo necessárias avaliações tanto a curto quanto a longo prazo.

O hipogonadismo secundário à deficiência de gonadotropinas é comum na fase aguda do TCE. Num estudo 12 dias após o trauma, Agha e cols. (21) mostraram uma prevalência de $80 \%$ de hipogonadismo. A longo prazo, a prevalência varia consideravelmente, 
de $2 \%$ a $32 \%(22,23)$. Enquanto na fase aguda após TCE o hipogonadismo se torna mais frequente quanto maior for a gravidade do trauma, a longo prazo não foi evidenciada tal correlação (10).

Clinicamente, além de seu efeito na libido e na fertilidade no sexo masculino, a deficiência de testosterona pode causar disfunção erétil, ginecomastia, apatia, redução da força muscular e da densidade óssea; nas mulheres hipogonádicas, ocorrem disfunções menstruais, labilidade emocional, fogachos, insônia, dispareunia e osteoporose.

Diversos fatores podem causar ou contribuir para a supressão do eixo gonadal após TCE. Injúria vascular, edema, hipóxia, hipertensão intracraniana ou injúria mecânica direta são possíveis mecanismos de dano à região hipotalâmico-hipofisária (24). A hiperprolactinemia, bem reconhecida por causar inibição do eixo hipotálamo-hipófise-gonadal, também tem sido relatada tanto na fase aguda quanto crônica do TCE $(21,25)$.

Nas mulheres pré-menopáusicas, o diagnóstico de hipogonadismo hipogonadotrófico é relativamente fácil, pois leva a alterações menstruais. Concentrações séricas de LH, FSH e estrogênio podem estar normais ou diminuídas, dependendo do grau de deficiência gonadotrófica (21). Já no hipogonadismo hipogonadotrófico masculino, a concentração sérica de testosterona estará diminuída com níveis de FSH e LH baixos ou normais.

$\mathrm{O}$ tratamento do hipopituitarismo envolve primeiramente a resolução da causa, quando for possível. Afastadas as contraindicações (câncer de próstata ou câncer de mama), a reposição hormonal deve ser iniciada no intuito de melhorar o estado de hipopituitarismo e suas consequências (18).

A avaliação do eixo hipofisário-gonadal de rotina nos sobreviventes TCE se faz necessária para o diagnóstico de deficiências hormonais. Entre essas deficiências, o hipogonadismo é reconhecido hoje como uma frequente complicação secundária ao TCE, contribuindo para a alta morbidade observada nesses pacientes. Assim, seu diagnóstico representa uma oportunidade para instituir um tratamento adequado com melhora clínica do déficit, além do bem-estar do paciente.

\section{MÉTODOS}

\section{Desenho do estudo}

Estudo descritivo prospectivo realizado com pacientes vítimas de traumatismo cranioencefálico grave na macrorregião de Florianópolis, atendidos no Hospital Go- vernador Celso Ramos (HGCR), que necessitaram de assistência médica na Unidade de Tratamento Intensivo. O estudo pertence ao projeto "Neurotrauma: bases fisiopatológicas, diagnóstico, fatores prognósticos e tratamento" aprovado no Comitê de Ética da Universidade Federal de Santa Catarina (UFSC) com o código 163/05.

\section{Tamanho da população}

A amostra estudada foi formada por 30 pacientes $(22$ homens e 8 mulheres), todos sobreviventes de TCE grave no período de 2000 a 2004. As informações dos pacientes constavam em um banco de dados do HGCR, hospital de referência na área de neurotraumatologia, na região da Grande Florianópolis. Neste banco de dados, constavam 176 pacientes que receberam alta após o TCE, porém, destes, apenas 95 pacientes foram encontrados e estavam vivos. A partir do grupo de 95 pacientes, foram utilizados critérios de inclusão e exclusão para o delineamento da população a ser estudada.

\section{Critérios de inclusão}

- Idade igual ou superior a 18 anos completos.

- Pacientes residentes da Grande Florianópolis.

- Pacientes que aceitaram a realização da pesquisa formalmente.

\section{Critérios de exclusão}

- Dificuldades de transporte.

- Pacientes institucionalizados.

- Não localização do paciente.

\section{Operacionalidade}

Os pacientes (ou responsáveis) selecionados assinaram o termo de consentimento informado e foram entrevistados pela equipe de Endocrinologia e Metabologia do Hospital Universitário da Universidade Federal de Santa Catarina (HU-UFSC), com a realização da anamnese e exame físico geral. A anamnese foi embasada em um questionário a fim de investigar possível sintomatologia relacionada à deficiência dos hormônios sexuais.

Todos os pacientes foram submetidos à avaliação laboratorial da função hipofisária por meio das seguintes dosagens: estradiol (mulheres), testosterona total (homens), LH, FSH e prolactina. Se alterados, os exames eram repetidos numa nova coleta. Foi feita uma média aritmética simples dos resultados alterados.

A coleta das amostras de sangue periférico foi realizada entre 8 e 10 horas da manhã, após jejum de 10 a 
12 horas, tendo sido retirados $10 \mathrm{~mL}$ de sangue através de punção venosa periférica com BD Vacutainer. Após coagulação, o sangue coletado foi centrifugado a 2.500 rotações por minuto $(\mathrm{rpm})$ durante 10 minutos. O sobrenadante foi separado e armazenado em tubos de ensaio à temperatura de $-20^{\circ} \mathrm{C}$ para dosagem hormonal posteriormente.

Utilizou-se o método enzimático de imunoquimioluminescência automatizada, com processamento em um analisador Immulite 2000 (Diagnostic Products Corporation - DPC) e um mesmo kit empregado para as dosagens de cada hormônio específico (Immulite ${ }^{\circledR}$ estradiol, Immulite ${ }^{\circledR}$ testosterona total, Immulite ${ }^{\circledR}$ FSH, Immulite ${ }^{\circledR}$ LH e Immulite ${ }^{\circledR}$ prolactina). As dosagens hormonais foram realizadas no Laboratório de Análises Clínicas do HU-UFSC.

Os resultados foram armazenados no programa SPSS (Statistical Software Program - versão 12.0) para serem analisados em conjunto posteriormente.

\section{Variáveis estudadas}

Foram coletadas as variáveis clínicas, demográficas e laboratoriais, incluindo idade, sexo, causa do TCE, escala de coma de Glasgow (ECG), classificação tomográfica de Marshall e tempo de avaliação após o TCE.

As dosagens hormonais realizadas foram testosterona total (para homens), estradiol (para mulheres), LH, FSH e prolactina (para ambos).

\section{Avaliação dos dados}

A análise estatística foi realizada utilizando-se o programa SPSS, versão 12.0, de 2003. Na análise estatística, o teste $t$ foi utilizado para os dados tipo contínuo, como para o valor de $\mathrm{LH}$, de FSH, idade média e tempo médio após TCE. Os dados nominais como sexo, escala de coma de Glasgow e classificação tomográfica de Marshall foram comparados por dois testes: teste exato de Fisher e o de qui-quadrado.

\section{Aspectos éticos}

O trabalho preenche os pressupostos éticos considerados necessários e suficientes pela "American Medical Association" (26) para considerar a pesquisa clínica proposta ética, incluindo valor da pesquisa para aumentar o conhecimento e/ou aprimorar a saúde, validade científica (rigor científico), ética na seleção dos casos, risco-benefício da execução do projeto favorável, revisão pelo Comitê de Ética, consentimento informado, respeito à privacidade e vontade dos pacientes envolvidos. Foi obtido termo de consentimento do paciente para a coleta das amostras de sangue.

\section{RESULTADOS}

Foram incluídos no estudo 30 pacientes, sendo 22 homens, entre 21 e 65 anos, com média de 38 anos, e 8 mulheres, de 18 a 75 anos, tendo em média 42 anos. O intervalo médio entre o TCE e a avaliação laboratorial foi de 4 anos. A idade média na época do traumatismo era de 33 e 37,5 anos para homens e mulheres, respectivamente.

As principais causas de traumatismo cranioencefálico encontradas foram acidente de trânsito $(63,4 \%)$, queda $(23,3 \%)$, agressão física $(6,7 \%)$, atropelamento por jet ski $(3,3 \%)$ e queda de árvore sobre este $(3,3 \%)$.

Tanto a faixa etária quanto as causas do TCE foram semelhantes entre o grupo total de 176 pacientes e o subgrupo de 30 pacientes estudados.

Entre os sintomas típicos de deficiência de hormônios sexuais, os mais prevalentes no sexo masculino foram disfunção erétil, diminuição da libido e fadiga, apresentados por 6 pacientes (27,3\% dos homens). No grupo feminino, 6 pacientes $(75 \%$ das mulheres) queixavam-se de labilidade emocional e 3 mulheres $(37,5 \%)$, de oligomenorreia e alterações do sono.

Foram encontrados 3 pacientes com níveis de FSH abaixo do normal, representando $10 \%$ do total, dos quais 2 eram homens $(66,7 \%)$ e 1 era mulher $(33,3 \%)$. Destes, 2 receberam escores entre 7 e 8 e o terceiro recebeu de 5 a 6 pontos segundo a ECG. Na classificação de Marshall, 2 pacientes possuíam injúria tipo III e 1 paciente apresentou lesão de massa evacuada. As idades dos homens eram 21 e 28 anos e da mulher era 20 anos. $\mathrm{O}$ tempo médio de avaliação desses 3 pacientes foi de 4,4 anos, semelhante ao dos pacientes com FSH inalterado $(4,6$ anos).

Em relação ao LH, apenas uma paciente, com idade de 20 anos, apresentou valor abaixo do normal, correspondendo a $3,3 \%$ da amostra (Tabela 2 ). No atendimento inicial, recebeu nota de 5 a 6 segundos a ECG. Na tomografia computadorizada de crânio, possuía lesão de massa evacuada de acordo com a classificação de Marshall. O tempo de avaliação após TCE foi de 3,1 anos.

Entre o grupo das mulheres, não foi observada alteração nos níveis de estradiol.

No grupo masculino, 2 pacientes $(9,1 \%)$ apresentavam níveis de testosterona abaixo do valor de referência $(<300 \mathrm{ng} / \mathrm{dL})$ (27). Possuíam valor médio de LH de 
$1,8 \mathrm{mIU} / \mathrm{mL}$, com desvio-padrão de $\pm 0,3 \mathrm{mIU} / \mathrm{mL}$. Esses pacientes receberam escores de 5 a 8 na ECG. Os achados tomográficos indicaram injúria tipo III em um desses pacientes e o outro apresentou lesão de massa evacuada. As idades dos pacientes com testosterona baixa eram 28 e 45 anos. A avaliação endocrinológica foi realizada em média 5,4 anos após o traumatismo, enquanto os demais pacientes masculinos foram avaliados em média 4,7 anos após o TCE.

Vinte pacientes apresentaram níveis de testosterona normal, correspondendo a 90,9\% dos homens (Tabela 3). Estes apresentaram $\mathrm{LH}$ em média de $3,7 \mathrm{mIU} / \mathrm{mL}$ com variação de $\pm 1,4 \mathrm{mIU} / \mathrm{mL}$. A metade dos pacientes com testosterona normal recebeu notas de 7 a 8 na ECG, 1 paciente $(5 \%)$ recebeu de 5 a 6 pontos e 9 pacientes $(45 \%)$ receberam menos de 5 pontos. De acordo com a classificação de Marshall, 5 pacientes (25\%) apresentaram injúria difusa tipo II, outros 6 pacientes $(30 \%)$ possuíram injúria tipo III, l paciente $(5 \%)$ apresentou injúria tipo IV e 8 pacientes (40\%) apresentaram lesão de massa evacuada. A idade média dos pacientes com testosterona normal foi de 31,6 anos, com variação de $\pm 13,9$ anos. Observou-se associação entre níveis baixos de testosterona e valores menores de LH $(\mathrm{p}=0,001)$.

\section{DISCUSSÃO}

Este estudo foi realizado por meio de um banco de dados do HGCR. O número de pacientes que sofreram TCE no período de 2000 a 2004 e que receberam alta desse hospital foi de 176. Destes, 95 pacientes foram localizados e apenas 30 deles preencheram os critérios necessários para a pesquisa. As principais limitações encontradas para localizar os pacientes foram mudança de residência, o fato de alguns pacientes terem sido institucionalizados e de outros não apresentarem condições de transporte, acarretando diminuição da amostra final, o que demonstra que o TCE leva a modificações no cotidiano, como perda da independência, desemprego, dificuldades financeiras, dificuldades de locomoção e prejuízo nas relações interpessoais. Semelhante ao que foi constatado por Edwards e Clark (28), houve prevalência maior de TCE em pacientes jovens masculinos, principalmente abaixo de 35 anos, o que contribui para que o TCE seja um problema de saúde pública, uma vez que a população de maior risco é aquela que se encontra em idade de maior produtividade.

Muitos dos pacientes questionados em nosso estudo afirmaram apresentar sintomatologia relacionada à deficiência de hormônios sexuais, apesar de apenas 9,1\%

\begin{tabular}{|c|c|c|c|c|}
\hline \multirow[b]{2}{*}{ Variável } & \multirow{2}{*}{$\begin{array}{c}\text { Todos } \\
\text { Pacientes } \\
\mathbf{n}=\mathbf{3 0}(\mathbf{1 0 0 \% )}\end{array}$} & \multicolumn{2}{|c|}{ LH } & \multirow[b]{2}{*}{ Valor de $\mathrm{p}^{\mathrm{a}}$} \\
\hline & & $\begin{array}{c}\text { Normal } \\
n=29(96,7 \%)\end{array}$ & $\begin{array}{c}\text { Baixo } \\
\mathrm{n}= \\
01(3,3 \%)\end{array}$ & \\
\hline \multicolumn{5}{|l|}{ Sexo } \\
\hline Masculino & $22(73,3)$ & $22(75,9)$ & 0 & \\
\hline Feminino & $8(26,7)$ & $7(24,1)$ & $1(100)$ & 0,23 \\
\hline \multicolumn{5}{|l|}{ ECG } \\
\hline Escore 7-8 & $14(46,7)$ & $14(48,3)$ & 0 & \\
\hline Escore 5-6 & $6(20,0)$ & $5(17,2)$ & $1(100)$ & \\
\hline Escore 3-4 & $10(33,3)$ & $10(34,5)$ & 0 & 0,12 \\
\hline \multicolumn{5}{|l|}{ Marshall } \\
\hline Injúria difusa tipo II & $5(16,7)$ & $5(17,3)$ & 0 & \\
\hline Injúria tipo III & $11(36,7)$ & $11(37,9)$ & 0 & \\
\hline Injúria tipo IV & $2(6,6)$ & $2(6,9)$ & 0 & \\
\hline Lesão de massa evacuada & $12(40,0)$ & $11(37,9)$ & $1(100)$ & 0,67 \\
\hline \multicolumn{5}{|l|}{ Idade } \\
\hline Média, em anos ( \pm DP) & & $35,6( \pm 15,7)$ & 20,0 & $0,40^{\mathrm{b}}$ \\
\hline $14-30$ anos & $16(53,3)$ & $15(51,7)$ & $1(100)$ & \\
\hline 31-45 anos & $9(30,0)$ & $9(31,0)$ & 0 & \\
\hline $46-60$ anos & $2(6,7)$ & $2(6,9)$ & 0 & \\
\hline$>60$ anos & $3(10,0)$ & $3(10,4)$ & 0 & \\
\hline \multicolumn{5}{|l|}{ Tempo após TCE } \\
\hline Média (DP) & & $4,6( \pm 1,4)$ & 3,1 & $0,30^{\mathrm{b}}$ \\
\hline
\end{tabular}

a Qui-quadrado ou teste exato de Fisher; ${ }^{\mathrm{b}}$ Teste $t$. 


\begin{tabular}{|c|c|c|c|c|}
\hline \multirow[b]{2}{*}{ Variável } & \multirow{2}{*}{$\begin{array}{c}\text { Todos } \\
\text { Pacientes } \\
\text { masculinos } \\
\mathbf{n = 2 2}(\mathbf{1 0 0 \% )}\end{array}$} & \multicolumn{2}{|c|}{ Testosterona } & \multirow[b]{2}{*}{ Valor de $\mathrm{p}^{\mathrm{a}}$} \\
\hline & & $\begin{array}{c}\text { Normal } \\
\mathrm{n}=\mathbf{2 0}(\mathbf{9 0 , 9 \% )}\end{array}$ & $\begin{array}{c}\text { Baixo } \\
n=2(9,1 \%)\end{array}$ & \\
\hline \multicolumn{5}{|l|}{ LH } \\
\hline Média (DP \pm ) & & $3,7(1,4)$ & $1,8(0,3)$ & $0,001^{b}$ \\
\hline Normal & $20(90,9)$ & $20(100)$ & 0 & \\
\hline Baixo & $2(9,1)$ & 0 & $2(100)$ & \\
\hline \multicolumn{5}{|l|}{ ECG } \\
\hline Escore 7-8 & $11(50,0)$ & $10(50,0)$ & $1(50,0)$ & \\
\hline Escore 5-6 & $2(9,1)$ & $1(5,0)$ & $1(50,0)$ & \\
\hline Escore 3-4 & $9(40,9)$ & $9(45,0)$ & 0 & 0,08 \\
\hline \multicolumn{5}{|l|}{ Marshall } \\
\hline Injúria difusa tipo II & $5(22,7)$ & $5(25,0)$ & 0 & \\
\hline Injúria tipo III & $7(31,8)$ & $6(30,0)$ & $1(50,0)$ & \\
\hline Injúria tipo IV & $1(4,6)$ & $1(5,0)$ & 0 & \\
\hline Lesão de massa evacuada & $9(40,9)$ & $8(40,0)$ & $1(50,0)$ & 0,83 \\
\hline \multicolumn{5}{|l|}{ Idade } \\
\hline Média, em anos (DP \pm ) & & $31,6( \pm 13,9)$ & $36,0( \pm 11,3)$ & $0,67^{b}$ \\
\hline $14-30$ anos & $12(54,5)$ & $11(55,0)$ & $1(50,0)$ & \\
\hline $31-45$ anos & $7(31,8)$ & $6(30,0)$ & $1(50,0)$ & \\
\hline $46-60$ anos & $2(9,1)$ & $2(10,0)$ & 0 & \\
\hline$>60$ anos & $1(4,6)$ & $1(5,0)$ & 0 & \\
\hline \multicolumn{5}{|l|}{ Tempo após TCE } \\
\hline Média (DP \pm ) & & $4,7( \pm 1,4)$ & $5,4( \pm 2,1)$ & $0,47^{b}$ \\
\hline
\end{tabular}

${ }^{\mathrm{a}}$ Qui-quadrado ou teste exato de Fisher; ${ }^{\mathrm{b}}$ Teste $t$.

dos pacientes masculinos serem diagnosticados com hipogonadismo. Os dois pacientes que apresentaram hipogonadismo laboratorial referiam queda de libido, sintoma com melhor relação de sensibilidade e especificidade no diagnóstico clínico de hipogonadismo no adulto (29). Outros estudos examinaram a frequência do hipogonadismo em pacientes sobreviventes de traumatismo cranioencefálico, que foram testados meses a anos após tal evento. A prevalência relatada alcançou de $2 \%$ a $30 \%$. No entanto, estudos mais recentes mostraram menor variação, de $9 \%$ a $17 \%(14,20,24,25)$, sendo mais compatível com o resultado do presente trabalho. Nos pacientes vítimas de TCE, a dosagem dos esteroides sexuais plasmáticos e dos níveis de gonadotropinas, associada a um contexto clínico, é suficiente para o diagnóstico de hipogonadismo central (2l). O diagnóstico de hipogonadismo em mulheres na prémenopausa é facilitado, uma vez que leva a alterações menstruais, sendo mais perceptível para a própria paciente a necessidade de buscar tratamento médico. No entanto, esse diagnóstico no grupo masculino pode ser mais complicado. Geralmente não apresenta alterações no exame físico, visto que a diminuição da pilificação e a ginecomastia são observadas posteriormente. Laboratorialmente, o hipogonadismo central masculino cursa com níveis baixos de testosterona na ausência de aumento de LH (21). Nas mulheres, encontra-se um nível de estradiol baixo na ausência de níveis de FSH elevados. Acredita-se, atualmente, que o hipogonadismo e a deficiência de hormônio de crescimento são as alterações hormonais mais comuns desses pacientes (21). É necessário, portanto, que os pacientes sejam avaliados e recebam seguimento a longo prazo a fim de detectar a ocorrência de hipopituitarismo pós-traumático.

As dosagens de prolactina encontravam-se normais em todos os pacientes, o que sugere integridade da haste hipofisária e do próprio hipotálamo. Klose e cols. (30) evidenciaram hiperprolactinemia em 39\% dos pacientes com TCE estudados. É importante a realização da sua dosagem, pois, quando aumentada, pode levar à supressão do eixo hipofisário-gonadal.

A classificação da escala de coma de Glasgow e a classificação de Marshall são importantes fatores na determinação do prognóstico dos pacientes com TCE. Todos os pacientes do estudo apresentaram TCE grave e, ao analisar os escores da ECG com as dosagens alte- 
radas de FSH, LH e testosterona, conclui-se não haver associação significativa entre estes para essa população. Isso sugere que pacientes com lesões mais graves e, portanto, prognóstico pior não desenvolveram tardiamente mais alterações dos hormônios estudados em relação aos pacientes com lesões menos graves. Ao contrário do presente estudo, Klose e cols. (22) evidenciaram risco aumentado de hipogonadismo pós-traumático em pacientes vítimas de TCE grave.

\section{CONCLUSÕES}

Este trabalho faz parte do primeiro estudo que avalia prospectivamente os casos de TCE grave na Grande Florianópolis (31). Também, de forma inédita, foram convocados pacientes de maneira tardia para fazer uma avaliação do eixo hipotálamo-hipófise-alvo, independente de apresentarem ou não qualquer sintomatologia.

$\mathrm{Na}$ Grande Florianópolis, foi constatado um maior número de casos de TCE em adultos jovens e do sexo masculino. Entre as causas de TCE, destacam-se os acidentes de trânsito, as quedas e as agressões físicas. No entanto, o grande responsável por TCE, no nosso meio, refere-se ainda aos acidentes de trânsito (31).

Recentemente, o hipopituitarismo tem sido reconhecido como uma possível complicação pós-traumática. O hipogonadismo é uma das alterações hipofisárias mais comuns nos pacientes vítimas de TCE, tendo sido observado em 9,1\% dos homens avaliados neste estudo. Na população feminina, entretanto, não foi encontrado nenhum caso de hipogonadismo. O tamanho reduzido da amostra feminina pode justificar esse achado no nosso estudo. Aimaretti e cols. (20) avaliaram 100 pacientes com TCE e encontraram hipogonadismo em 14 homens e em 3 mulheres. Já no estudo de Agha e cols. (21), de um universo de 102 pacientes estudados, 12 pacientes apresentaram deficiência de gonadotropinas e, destes, apenas 2 eram mulheres.

Deve-se, portanto, considerar o hipogonadismo como um déficit hormonal possível nos sobreviventes de TCE, especialmente em adultos jovens do sexo masculino. Desse modo, uma avaliação rotineira do eixo hipofisário-gonadal nos pacientes de maior risco deveria ser instituída, uma vez que o tratamento visa otimizar a recuperação e melhorar a qualidade de vida destes.

Agradecimentos: este trabalho obteve apoio do Conselho Nacional de Desenvolvimento Científico e Tecnológico (CNPq) e da Fundação de Amparo à Pesquisa do Estado de Santa Catarina (Fapesc).
Declaração: os autores declaram não haver conflitos de interesse científico neste estudo.

\section{REFERÊNCIAS}

1. Khan F, Baguley IJ, Cameron ID. 4: Rehabilitation after traumatic brain injury. Med J Aust. 2003;178(6):290-5.

2. Salazar AM, Warden DL, Schwab K, Spector J, Braverman S, Walter $\mathrm{J}$, et al. Cognitive rehabilitation for traumatic brain injury: a randomized trial. Defense and Veterans Head Injury Program (DVHIP) Study Group. JAMA. 2000;283(23):3075-81.

3. Schwarzbold M, Diaz A, Martins ET, Rufino A, Amante LN, Thais $M E$, et al. Psychiatric disorders and traumatic brain injury. Neuropsychiatr Dis Treat. 2008;4(4):797-816.

4. Rehabilitation of persons with traumatic brain injury. NIH Consens Statement. 1998;16(1):1-41.

5. Bruns J Jr, Hauser WA. The epidemiology of traumatic brain injury: a review. Epilepsia. 2003;44 Suppl 10:2-10.

6. Masini M. Perfil epidemiológico do traumatismo cranioencefálico no Distrito Federal em 1991. J Bras Neurocirurgia. 1994;5:61-8.

7. van Baalen B, Odding E, Maas Al, Ribbers GM, Bergen MP, Stam HJ. Traumatic brain injury: classification of initial severity and determination of functional outcome. Disabil Rehabil. 2003;25(1):9-18.

8. KalsbeekWD, McLaurin RL, Harris BS, 3rd, Miller JD. The National Head and Spinal Cord Injury Survey: major findings. J Neurosurg. 1980;Suppl:S19-31.

9. Koizumi MS, Lebrao ML, Mello-Jorge MH, Primerano V. Morbidity and mortality due to traumatic brain injury in Sao Paulo City, Brazil, 1997. Arq Neuropsiquiatr. 2000;58(1):81-9.

10. Agha A, Thompson CJ. High risk of hypogonadism after traumatic brain injury: clinical implications. Pituitary. 2005;8(3-4):245-9.

11. Kimura A. The current status and problems of rehabilitation for TBI (traumatic brain injury) patients in Japan. Keio $\mathrm{J}$ Med. 2003;52(2):100-6.

12. Masel BE. Rehabilitation and hypopituitarism after traumatic brain injury. Growth Horm IGF Res. 2004;14 Suppl A:S108-13.

13. Lamberts SW, de Herder WW, van der Lely AJ. Pituitary insufficiency. Lancet. 1998;352(9122):127-34.

14. Lieberman SA, Oberoi AL, Gilkison CR, Masel BE, Urban RJ. Prevalence of neuroendocrine dysfunction in patients recovering from traumatic brain injury. J Clin Endocrinol Metab. 2001;86(6):2752-6.

15. Elovic EP, Glenn MB. Anterior pituitary dysfunction after traumatic brain injury, part II. J Head Trauma Rehabil. 2004;19(2):184-7.

16. Popovic V, Aimaretti G, Casanueva FF, Ghigo E. Hypopituitarism following traumatic brain injury (TBI): call for attention. J Endocrinol Invest. 2005;28(5 Suppl):61-4.

17. Bondanelli $M$, Ambrosio $M R$, Zatelli $M C$, De Marinis $L$, degli Uberti EC. Hypopituitarism after traumatic brain injury. Eur J Endocrinol. 2005;152(5):679-91.

18. Leal-Cerro A, Flores JM, Rincon M, Murillo F, Pujol M, GarciaPesquera $F$, et al. Prevalence of hypopituitarism and growth hormone deficiency in adults long-term after severe traumatic brain injury. Clin Endocrinol (Oxf). 2005;62(5):525-32.

19. Kelly DF, Gonzalo IT, Cohan P, Berman N, Swerdloff R, Wang C. Hypopituitarism following traumatic brain injury and aneurysmal subarachnoid hemorrhage: a preliminary report. J Neurosurg. 2000;93(5):743-52.

20. Aimaretti G, Ambrosio MR, Di Somma C, Fusco A, Cannavo S, Gasperi $M$, et al. Traumatic brain injury and subarachnoid haemorrhage are conditions at high risk for hypopituitarism: screening study at 3 months after the brain injury. Clin Endocrinol (Oxf). 2004;61(3):320-6. 
21. Agha A, Rogers B, Sherlock M, O'Kelly P, Tormey W, Phillips J, et al. Anterior pituitary dysfunction in survivors of traumatic brain injury. J Clin Endocrinol Metab. 2004;89(10):4929-36.

22. Klose M, Juul A, Poulsgaard L, Kosteljanetz M, Brennum J, FeldtRasmussen U. Prevalence and predictive factors of post-traumatic hypopituitarism. Clin Endocrinol (Oxf). 2007;67(2):193-201.

23. Schneider HJ, Schneider M, Saller B, Petersenn S, Uhr M, Husemann $B$, et al. Prevalence of anterior pituitary insufficiency 3 and 12 months after traumatic brain injury. Eur J Endocrinol. 2006;154(2):259-65.

24. Benvenga S, Campenni A, Ruggeri RM, Trimarchi F. Clinical review 113: Hypopituitarism secondary to head trauma. J Clin Endocrinol Metab. 2000;85(4):1353-61.

25. Agha A, Rogers B, Mylotte D, Taleb F, Tormey W, Phillips J, et al. Neuroendocrine dysfunction in the acute phase of traumatic brain injury. Clin Endocrinol (Oxf). 2004;60(5):584-91.

26. Emanuel L. Building in ethics at the American Medical Association: a case study in purpose, structure and management. Qual Manag Health Care. 2000;8(3):1-13.
27. Bhasin S, Cunningham GR, Hayes FJ, Matsumoto AM, Snyder PJ, Swerdloff RS, et al. Testosterone therapy in adult men with androgen deficiency syndromes: an endocrine society clinical practice guideline. J Clin Endocrinol Metab. 2006;91(6):1995-2010.

28. Edwards OM, Clark JD. Post-traumatic hypopituitarism. Six cases and a review of the literature. Medicine (Baltimore). 1986;65(5):281-90.

29. Clapauch R, Braga DJ, Marinheiro LP, Buksman S, SchrankY. Risk of late-onset hypogonadism (andropause) in Brazilian men over 50 years of age with osteoporosis: usefulness of screening questionnaires. Arq Bras Endocrinol Metabol. 2008;52(9):1439-47.

30. Klose M, Juul A, Struck J, Morgenthaler NG, Kosteljanetz M, Feldt-Rasmussen $U$. Acute and long-term pituitary insufficiency in traumatic brain injury: a prospective single-centre study. Clin Endocrinol (Oxf). 2007;67(4):598-606.

31. Martins ET, Linhares MN, Sousa DS, Schroeder HK, Meinerz J, Rigo LA, et al. Mortality in severe traumatic brain injury: a multivariated analysis of 748 Brazilian patients from Florianopolis City. JTrauma. 2009;67(1):85-90. 